\title{
La culture ordinaire, une heuristique des STS pour former à la médiation culturelle des sciences et techniques en société
}

\section{Michel Letté}

\section{OpenEdition}

\section{Journals}

Édition électronique

URL : http://journals.openedition.org/trema/3934

DOI : 10.4000/trema.3934

ISSN : 2107-0997

Éditeur

Faculté d'Éducation de l'université de Montpellier

Édition imprimée

Date de publication : 1 mars 2018

Pagination : 75 - 96

ISBN : 979-10-96627-04-2

ISSN : 1167-315X

\section{Référence électronique}

Michel Letté, "La culture ordinaire, une heuristique des STS pour former à la médiation culturelle des sciences et techniques en société », Tréma [En ligne], 48 | 2018, mis en ligne le 01 juin 2018, consulté le 30 avril 2019. URL : http://journals.openedition.org/trema/3934 ; DOI : 10.4000/trema.3934

Ce document a été généré automatiquement le 30 avril 2019.

Trema 


\title{
La culture ordinaire, une heuristique des STS pour former à la médiation culturelle des sciences et techniques en société
}

\author{
Michel Letté
}

\section{Introduction}

1 Il y a un peu plus de 10 ans, un nouvel enseignement dédié à la culture des sciences et techniques voyait le jour au Conservatoire national des arts et métiers. Destiné aux professionnels des relations publiques et de la communication des organismes de recherche, il venait à l'appui des politiques de gestion de l'innovation sous l'égide de ladite gouvernance. Complétant des formations plus anciennes de cet établissement, il participait de cette façon à l'une de ses missions, celle de contribuer à la diffusion de la culture et à la valorisation du patrimoine scientifique et technique ${ }^{1}$.

L'ancrage social et historique, comme les motifs économiques et politiques de ce nouvel enseignement n'étaient pas ignorés ${ }^{2}$. Ils rendaient compte du besoin d'adhésion des publics à l'édification d'une économie de la connaissance telle qu'énoncée en 2000 par la stratégie dite de Lisbonne ${ }^{3}$. La présidence du Conseil européen associait ainsi dans ses conclusions le terme «culture» respectivement et dans cet ordre aux adjectifs "d'entreprise», "technologique» et "numérique »". Elle témoignait d'une évolution toujours en cours des objectifs traditionnels de la culture scientifique et technique qui ne sont plus seulement de faire aimer la science pour elle-même ou de transmettre des contenus de connaissances expertes auprès d'un large public, mais de travailler aussi et surtout à la formation conjointe d'une économie de la connaissance et d'un public de citoyens éclairés sur ses enjeux. Il s'agit désormais de mettre en politique par la culture et la formation la question autrement plus complexe du rôle des technosciences dans la relance de la croissance économique en Europe ${ }^{5}$. 
3 Ce qui suppose de considérer autrement les publics visés, non plus seulement comme des réceptacles passifs de savoirs vulgarisés mais comme les agents sociaux d'une économie de la connaissance à construire ${ }^{6}$. Société de la connaissance et dialogue civil ont partie liée. La question de la fabrication conjointe des technosciences et de la société est ainsi devenue cruciale, mais aussi conflictuelle. Le débat public comme la controverse sociotechnique se sont banalisés ${ }^{7}$. Les innovations technoscientifiques sont devenues des objets de conflits 8 . Les décisions dans ces domaines sont désormais comprises par les publics comme autant de choix de société9. Ils invoquent les risques, les menaces pesant sur l'environnement et la santé, voire revendiquent l'édification d'une démocratie technique et environnementale ${ }^{10}$. Les formes du dialogue évoluent en conséquence autour de ces questions entre conflits et compromis ${ }^{11}$. Se recompose de cette façon tout l'assemblage des sociétés humaines et des technosciences dont les évolutions en matière de diffusion de la culture scientifique et technique est l'une des traductions. De plus en plus de ses acteurs sont ainsi appelés à se tourner vers l'animation de débats publics afin de considérer ces questions dites socialement vives ${ }^{12}$.

4 Si l'objectif des formations destinées aux médiateur-rice-s reste d'acquérir des savoirs et des savoir-faire pour la conception, la réalisation et l'animation de dispositifs de médiation culturelle des sciences et techniques, il s'agit désormais d'adjoindre à cette façon de dire les choses le terme "en société ». Les Sciences et Techniques en Société - ou Science and Technology Studies (STS) en anglais - rassemblent les travaux qui cherchent à expliciter la fabrication conjointe des sciences, des techniques et des sociétés ${ }^{13}$. Cette dimension STS de la culture scientifique et technique n'est plus ignorée. Dans le droit fil de la transformation conjointe des technosciences et de la démocratie, la formation des médiateur-rice-s est repensée depuis plusieurs années en Europe comme en France, de même ses ambitions comme ses manières de faire pédagogiques ${ }^{14}$. La culture STS est devenue un objet politique ${ }^{15}$. Les STS s'affirment de leur côté comme un objet des politiques culturelles. D'où l'impératif pour la formation d'une initiation à ce vaste champ de la pensée et de l'action afin que les étudiant-e-s saisissent le contexte dans lequel ils/ elles exercent leurs activités. Ils/elles acquièrent de cette façon les outils conceptuels et d'analyse leur permettant de s'approprier les exigences de leurs commanditaires, parfois pour les retravailler ou les renégocier. Ce qui pose certes la question du comment technique, mais surtout celle du pourquoi politique. La question impose en tout cas une lecture plus STS des objectifs comme des moyens mis en œuvre pour la médiation des sciences et techniques, singulièrement des technosciences ${ }^{16}$.

5 La traduction de ces évolutions en contenus d'enseignement ne va cependant pas de soi. Emprunter des chemins de traverse est indispensable. La difficulté est réelle de soumettre les étudiant-e-s à l'épreuve de la seule littérature des STS. Ce serait les confronter trop rapidement à ce qu'ils/elles pensent ou font déjà en matière de diffusion de la culture scientifique et technique. Si un cours d'initiation aux STS leur est dispensé par ailleurs, une approche complémentaire est nécessaire afin de les inviter à les utiliser comme des outils d'analyse des relations entre sciences, techniques, cultures et société, mais aussi d'analyse de leurs propres relations avec les publics auxquels ils/elles s'adressent. C'est l'objet de cet article de rendre compte d'une expérience les invitant à s'emparer des problématiques STS en lien avec leurs motivations, leurs conceptions des pratiques de la médiation comme le sens donné à leurs activités présentes et à venir. De ce point de vue, il convient de partir de ce que sont et pensent les élèves en début de parcours afin 
d'engager une démarche pédagogique adaptée, le but étant de leur permettre d'investir rapidement le champ des STS comme partie intégrante de leur formation.

Quelles sont leurs attentes et représentations des fonctions de la médiation culturelle des sciences et techniques comme des publics auxquels ils/elles s'adressent ? C'est l'objet de la première partie de préciser les raisons d'une adéquation entre les attentes des étudiant-e-s et les attendus de la formation. Afin de cerner les cadres dominants de pensée et d'action en matière de médiation, ils/elles renseignent un questionnaire lors de la première séance de cours afin de recueillir leurs conceptions spontanées de ce que sont la médiation et ses publics. Cette façon de procéder permet de situer le spectre des épistémologies et valeurs auxquelles se réfèrent les élèves, mais aussi de mesurer les évolutions tout au long de leur parcours.

7 La seconde partie explicite l'hypothèse de travail mise à l'épreuve de la démarche pédagogique. Elle pose que les sciences et les techniques (industrie, technologie, technoscience, invention, découverte, connaissance savante, innovation, expertise, etc.) saturent l'espace public sous des formes les plus triviales, banales et parfois anodines, mais jamais sans conséquences sur la formation d'un imaginaire sur ce que les sciences et techniques font à la société, et réciproquement. La production et la consommation culturelle de masse, comme la culture dite populaire et l'expérience commune, sont pleines des manifestations de cette intrication des sciences, des techniques et de la société. Toutes ces manifestations forment le vaste champ de la culture ordinaire des STS, c'est-à-dire l'ensemble des médiations informelles par lesquelles les liens entre sciences, techniques et société se donnent à voir au travers des représentations populaires, du quotidien et du vécu. Ce sont ces questions pleines de choix de société dont témoigne la culture ordinaire. Elle n'est donc pas que l'expression d'une irrationalité de publics sans facultés de jugement. Elle est en réalité le lieu d'une médiation informelle et permanente des STS. Elle est une voie d'entrée dans l'univers foisonnant des imaginaires sociaux et politiques où la science et la technique tiennent le premier rôle. Autrement dit, ce qui est en jeu n'est ni la science ni la technique pour elle-même mais leurs traductions sociales. Science et technique n'ont donc pas attendu l'intervention publique pour exister culturellement, elles sont là toujours présentes, contenues dans l'ordinaire culturel de la production et de la consommation de masse. C'est à ce registre d'existence socioculturelle des sciences et techniques auquel les étudiant-e-s sont familiarisé-e-s. L'exploration de la culture ordinaire s'avère être en quelque sorte une heuristique qui leur permet de s'emparer des logiques de questionnement STS. Elle est ainsi mobilisée à la fois comme espace, matériau, ressource et ultimement comme voie d'une transition pédagogique afin que les étudiant-e-s considèrent les problématiques STS en lien direct avec leurs pratiques.

\section{Concilier attentes des étudiants et attendus de la formation}

\section{1. Des profils hétérogènes}

Qui sont les étudiant-e-s des formations du CNAM dédiées à la médiation culturelle des sciences et techniques? Les promotions sont chaque année composées d'environ 70 élèves si on inclut l'ensemble des inscrit-e-s, quel que soit l'enseignement spécifique suivi, le titre, le diplôme visé ou la progression dans le parcours, le mode de suivi des 
cours, en présentiel ou à distance (un individu peut suivre les enseignements sur une même année ou plusieurs, combiner présence et distance $)^{17}$. Un peu plus d'une vingtaine d'inscrit-e-s est ainsi nouvellement intégrée chaque année. Les promotions sont majoritairement composées de femmes avec une proportion d'hommes comprise entre 15 et $35 \%$ selon les années. L'une des caractéristiques principales de ces promotions est qu'elles réunissent une population hétérogène, riche d'une culture plurielle. Il faut selon les années travailler, par exemple, avec des individus issus de domaines aussi différents que sont le journalisme scientifique et l'architecture, l'histoire de l'art et la recherche en physique des matériaux, le design et la communication institutionnelle, la restauration des œuvres et l'enseignement secondaire. Il faut aussi concilier les divergences de vues générationnelles entre celles et ceux tout juste sorti-e-s de l'université muni-e-s d'une licence et des praticiens plus âgés en situation de réorientation professionnelle. Il faut rapprocher les situations de découverte d'un secteur d'activité pour les uns, et celles d'une quête de certification des compétences acquises pour les autres. Enfin, il faut assurer les conditions d'un échange entre les personnes à la recherche d'un emploi et les autres travaillant déjà à temps plein. Les situations familiales comme les disponibilités conséquentes sont tout aussi variables et variées, comme pour la plupart des auditeurrice-s de cet établissement d'ailleurs, dont la vocation est la formation continue, principalement en cours du soir.

9 La diversité des origines de formation, des situations personnelles comme des projets professionnels est toutefois compensée par le partage d'une vision relativement commune sur la nature des objectifs de la médiation et des relations à instaurer avec les publics. Elle transparaît nettement dans les lettres de motivation que les candidat-e-s à l'intégration de ces formations envoient avec leur curriculum vitae. Afin cependant de cerner mieux encore le cadre général de pensée en matière de médiation culturelle des sciences et techniques, un questionnaire papier leur est soumis dès la première séance de cours.

\section{2. Cerner les épistémologies dominantes}

En un temps relativement court, une trentaine d'élèves répond aux questions suivantes :

11 - Décrivez la médiation culturelle des sciences et techniques telle que vous la concevez (vocation, publics, objectifs, etc.);

12 - Citez (et décrivez au besoin) un ou 2 dispositifs qui vous semble(nt) parmi les plus pertinents ;

13 - Avez-vous des critiques à faire à l'encontre des dispositifs actuels de diffusion de la culture scientifique et technique?

14 - Décrivez ce que serait le dispositif idéal selon vous ;

15 - Quel(le) médiateur-rice serez-vous? Imaginez où et comment vous exercerez votre métier.

La première question est celle pour laquelle le plus d'éléments de réponse sont recueillis. Les suivantes ont surtout pour vocation à permettre à celles et ceux qui pratiquent déjà la médiation d'une façon ou d'une autre d'aller plus loin, de préciser leur propos. Ce questionnaire est donc renseigné de façon inégale, mais la première question comporte toujours une proposition, parfois sous la forme d'un seul mot, généralement un verbe (du type transmettre, vulgariser, communiquer). Si certain-e-s sont très prolixes et vont 
jusqu'à répondre aux questions qui ne leur ont pas été posées, notamment sous la forme de témoignages d'expériences vécues, d'autres sont plutôt économes, laissant sans réponses les dernières parties du questionnaire. La majorité consent cependant à mentionner des éléments pour chacune d'elles. Quelques réponses difficilement classables vous emmènent par exemple dans les sphères hautes de la poésie, telle cette médiatrice se qualifiant avant tout de « chercheuse d'émotions » et dont le dispositif idéal permettrait « d'emmener les gens dans l'espace ».

17 Ce questionnaire a été rempli durant trois années consécutives. Ce qui permet de disposer d'une centaine de réponses écrites. A cela s'ajoute quelques dizaines de retours supplémentaires de la part des élèves suivant cet enseignement à distance. A ce stade, il n'est évident ni de qualifier, ni de quantifier précisément ces données éparses produites sur papier libre, mais il est possible de les classer à partir demots clés, phrases et expressions selon le sens perçu par l'enseignant. Trois catégories homogènes de réponses s'en dégagent, dont la proportion est approximativement de 60/20/20.

Ce qui suit cite autant que possible les étudiant-e-s, retraduit, reformule et synthétise ce qu'ils/elles affirment au travers des échanges engagés après la remise du questionnaire afin de permettre à chacun-e de développer et de préciser sa pensée.

Une épistémologie dominante s'en dégage.

\section{3. Une science pour tous}

20 La première catégorie concerne près des $2 / 3$ de l'échantillon. Elle rassemble une façon similaire de dire la vocation première de la médiation, centrée sur le principe de la diffusion d'une culture scientifique et technique au singulier. Il s'agit d'abord de "divulguer des contenus de savoirs" sous une forme "accessible à un large public ». La médiation comme objectif de vulgarisation est ainsi clairement affirmée. Il faut «transmettre et vulgariser », « rendre accessible la connaissance », « faire comprendre la science» ou encore "expliquer simplement comment ça marche». La vocation de la médiation est alors de «faire le tri dans la masse des informations». Elle est de servir de "lien », de " pont », de " passerelle ", "d'interface » et de " point de rencontre » entre "la communauté des chercheurs et les jeunes publics", entre "les connaissances scientifiques et les croyances populaires", pour enfin "combler le fossé d'incompréhension entre scientifiques et publics ", "faciliter la communication » et "éduquer les populations à la démarche scientifique», voire même "de justifier, de rassurer, de répondre aux attaques et inquiétudes du public ». Parmi les réponses de cette nature, quelques-unes précisent la nécessité sous-jacente de "faire aimer la science», "d'éveiller la curiosité » et "l'intérêt », «de susciter des questions ", d'» amener les publics à s'interroger », tout en devant rester « neutre ».

21 Les lieux et moments associés à ces fonctions de la médiation telle qu'évoquer au travers de ces quelques mots sont avec «la fête de la science» les organismes traditionnels de diffusion de la culture scientifique et technique, tels la Cité des sciences et de l'industrie, le Palais de la découverte, les observatoires, les centres de culture scientifique et technique et industrielle, mais aussi les museums d'histoire naturelle. Les supports de diffusion désignés sont essentiellement les collections, les expositions et les conférences scientifiques. L'idéal pour les auteur-e-s de cette catégorie de réponses est d'exercer leur métier dans ces institutions. 


\section{4. Des sciences pour chacune et chacun}

«Selon moi la médiation culturelle des sciences et techniques sert à transmettre un savoir, une information, à vulgariser ou approfondir des concepts et des connaissances de manière sérieuse ou ludique (en faisant participer le public par exemple). Mais elle sert aussi à rendre les gens curieux, à se poser des questions sur le monde et les choses qui les entourent, le fonctionnement des machines, des êtres. De faire appel à leur sens critique et à leur réflexion, ouvrir le dialogue ».

Invitant finalement au dialogue, ce type de réponse mène à la catégorie suivante. Y sont classées les réponses renvoyant à un spectre plus large de la médiation au sein de laquelle la pluralité et la diversité des savoirs comme des publics ont un peu plus leur place. La question des frontières entre savants et profanes est abordée pour en discuter les rigidités, la nécessité de les bousculer ou du moins de les remettre parfois en question, sans pour autant devoir sombrer dans un relativisme sans fond où tous les savoirs se vaudraient. La production de connaissances scientifiques reste première pour consolider la voie d'une explication rationnelle du monde. Les notions de "partage», de « dialogue », d'» échanges », de " projet commun » et de «participation » accompagnent la description d'un champ de la médiation où les protagonistes ne se limitent pas aux seuls scientifiques et publics mais à des "communautés de savoirs", au " gouvernement », aux « industriels », aux « militaires », aux " politiques ». Il est question de "confrontation des points de vue», "d'ouverture des esprits", de "partage des expériences », de " développer sa propre culture des sciences et techniques », " d'avancer ensemble ». Pour cela il est nécessaire de créer des « espaces de dialogue et de respect mutuel », "de mettre en place de véritables espaces de vrais dialogues " permettant de "valoriser la parole des publics » et où "le public se sent à l'aise", de discuter "de nouvelles idées sur la science». Dans ce cas, le cadre d'exercice et de pratiques pertinentes est le milieu plutôt associatif, tel les « Petits débrouillards » ou les structures du type « Fab' Lab'».

\section{5. Mettre les sciences en politique}

La troisième catégorie, minoritaire comme la précédente, élargie encore un peu plus le champ de la médiation en incluant dans son périmètre une plus grande variété d'acteurs encore, de lieux et de pratiques. Les « réseaux sociaux », les « médias » et «internet » côtoient les "associations de sciences citoyennes ", le "tiers secteur scientifique », les « associations de patients et de malades » et les « ateliers collaboratifs et participatifs ». Il n'est plus ici seulement question de transmission de connaissances, mais de considérer "les impacts sur la société ", «les enjeux économiques", « les problèmes posés par les résultats de la recherche » au travers de « débats publics ». Il est question « d'autoriser la légitimité de la prise de parole des publics", "de décloisonner les frontières afin de mettre la science en démocratie » ou de "démocratiser les sciences», de "permettre l'écoute des publics auprès des décideurs", de "se mettre à distance critique face à l'image d'une science pure », de " rendre accessible les enjeux sociétaux », « d'instaurer le dialogue parmi les publics concernant les questions sociotechniques et environnementales ». Dans ces lignes transparaissent un vocabulaire plus sociopolitique, témoignage d'un passage initial par les sciences humaines et sociales (sociologie et histoire principalement), d'une conscience des enjeux portés par les « innovations » et de la nécessité de débattre des choix de société qu'ils impliquent. Si cette catégorie d'élèves 
n'évoque pas explicitement les STS, ses thématiques sont pourtant bien présentes en filigrane dans la formulation de leurs réponses. Elle est la plus à même d'accueillir comme une évidence l'impératif STS tout en étant en attente explicite d'éclairages sur les intrications complexes de la production scientifique et technique et l'organisation sociale et politique du monde.

\section{6. Prégnance toujours du déficit model}

La remise du questionnaire et les échanges collectifs qui suivent permettent de cerner la façon dont est pensée, voire déjà pratiquée, la médiation parmi les élèves. Dans leur grande majorité, ils adhèrent au modèle de l'instruction publique et de l'éducation populaire mais sans vraiment l'interroger. Leur vision est celle d'un monde rationnel toujours à construire où doit régner la distinction nette entre une vérité produite par les sciences et les opinions informes, entre le vrai scientifique et le sens commun des croyances populaires. Leurs conceptions des fonctions du médiateur sont d'abord celles d'un devoir de transmission des connaissances rigoureuses et robustes auprès de publics jugés ignorants, auxquels il est demandé de bien vouloir consentir à savoir. Le rôle du médiateur est alors de corriger les opinions approximatives à l'appui de la production rationnelle des sciences et techniques. Tout est affaire de transmission de savoirs experts, de ceux permettant de trancher les débats et les controverses, de faire la part scientifique des certitudes et des incertitudes. Il s'agit dans ce cas de pourvoir aux besoins d'une communication efficace et performante par la culture en direction de publics réputés animés par l'émotion et l'irrationnel.

L'un des présupposés sous-jacent à cette lecture du champ de la médiation est l'évidence que la culture scientifique et technique émane d'abord sinon exclusivement, de la connaissance produite par les savants et les experts eux-mêmes. La démarcation entre scientifiques et publics à instruire en est la conséquence logique. Entre les deux, un espace tampon est peuplé de médiateurs, eux-mêmes équipés de dispositifs et menant des opérations de médiation. Ils travaillent pour le compte des institutions traditionnelles de la culture prescrite (les musées et le vaste réseau des centres de la culture scientifique, technique et industrielle) et de plus en plus aujourd'hui pour toutes ces organisations ayant besoin de communiquer afin de justifier leur existence et leurs actions (entreprises, associations, collectifs, organismes publics de recherche, think tank et autres entités du lobbying). En conséquence, la médiation vise selon les cas la communication, le dialogue, la conciliation, l'échange, la traduction, l'intercession, la pacification, l'acceptation ... bref, assure toutes ces fonctions d'interfaçage, de mise en relation autant que possible sereine et sympathique d'univers réputés ne pas se comprendre. Le médiateur est alors tour à tour et selon les circonstances un représentant, négociateur, agent, mandataire, entremetteur, ambassadeur, animateur, missionnaire, conciliateur, interprète, arbitre, émissaire, diplomate, procureur, et parfois même un peu commerçant. Il doit disposer pour cela de toutes les compétences, de celles qui lui permettent d'être en situation de proposer ses dispositifs de médiation, de varier les supports et les contenus pour une mise en relation optimale de tous les publics avec les producteurs autorisés de savoirs scientifiques et les praticiens de la performance technique. Le médiateur se fait alors un peu sergent instructeur, chargé de «faire comprendre et aimer les sciences ». Il est en quelque sorte négociant en traduction et vulgarisation ${ }^{18}$. 
27 En forçant un peu le trait, disons que c'est à partir de cette vision antagoniste d'une science vraie à promouvoir et des opinions fausses à combattre qu'est essentiellement pensé le rôle du médiateur et la fonction sociopolitique de la médiation, même si la nuance est portée par la légitimité du débat public sur les sujets les plus controversés, légitimité globalement acquise parmi les élèves si elle s'appuie sur la parole du scientifique. Ils se considèrent dès lors non plus comme appartenant au monde des publics, mais comme les alliés des producteurs de connaissances scientifiques, voire euxmêmes comme des contributeurs à l'élaboration de savoirs. Comme la plupart des scientifiques, ingénieurs et techniciens dont les étudiant-e-s se font en quelque sorte les porte-paroles, ils/elles estiment n'avoir finalement que peu à apprendre des publics.

\section{La culture ordinaire, une heuristique des STS}

\section{1. Les STS selon les publics}

Cette conception d'une culture à inculquer aux publics ne conçoit pas, peu ou mal la nécessité en retour d'entendre ce que ces derniers peuvent avoir à dire et à penser. Pourtant ils s'expriment. Ils ont même tendance à se mêler de plus en plus de ce qui les regarde: environnement, santé, sécurité au travail, expertise, liberté individuelle et collective, financement de la recherche, impacts sociaux des technosciences, bioéthique, ... parmi de nombreux registres d'interrogations sur ce que les sciences et techniques font à la société, et réciproquement ${ }^{19}$.

Les médiateur-rice-s ne peuvent l'ignorer. Car il n'est en réalité plus une connaissance ou une pratique d'ordre scientifique ou technique qui échappe à la question de ses intrications avec le politique et le social, à celle de ses rapports avec la recherche, la production industrielle, l'innovation, l'économie, la finance, l'entreprise, la guerre, l'environnement, l'éthique, la démocratie, ... et finalement de tout ce qui relève de l'organisation politique du monde ${ }^{20}$. C'est à cette réalité que renvoie l'émergence des questions socialement vives au sortir des Trente glorieuses ${ }^{21}$. Les publics sont visiblement sensibles aux changements qui s'opèrent, et en premier lieu dans leur quotidien ${ }^{22}$.

30 Avec les technosciences, la culture scientifique et technique a progressivement investi le champ plus politique de la médiation. Ce dernier terme tend en effet à remplacer celui de "diffusion» de ladite culture scientifique, technique et industrielle qui reconsidère depuis ses façons de faire, suivant en cela les mutations socioéconomiques et politiques, et notamment la revendication d'une part croissante des publics de ne plus être seulement considérés comme les destinataires passifs de savoirs vulgarisés, mais d'être aussi entendus au travers de leurs opinions vulgaires ${ }^{23}$. D'autres types de prescriptions ont ainsi vu le jour, insistant sur les nécessaires « dialogues » et " participations » des publics, jusqu'aux incantations pour une culture partagée au travers d'une coconstruction des savoirs $^{24}$. Ce qui incite à l'écoute de ce qui se dit et se passe aussi du côté des publics.

31 La médiation culturelle des STS s'est de toute façon imposée, y compris dans le champ de l'intervention institutionnelle ${ }^{25}$. Et la façon de dire les choses rend compte de ces évolutions. Outre-Manche, cela s'est manifesté dans les années 2000 par l'emploi de l'expression public engagement with science and technology qui remplace progressivement celle de public understanding of science and technology. Sa vocation est d'impliquer autrement les publics ${ }^{26}$. En France, l'usage du terme « médiation » comporte 
le projet sinon de partage, au moins de conciliation par le dialogue et l'échange entre des ordres de pratiques et de savoirs différents ${ }^{27}$. Un autre point de vue dès lors s'impose. Il s'agit moins de focaliser l'attention sur le déficit des connaissances parmi les publics que sur ce qu'ils pensent de l'intrication des technosciences et de la société. Cet intérêt des publics pour les sciences et techniques en société (et non plus science et société) a contribué en partie à infléchir les pratiques traditionnelles en matière d'action culturelle. L'ambition s'est imposée peu à peu de considérer une revendication citoyenne de dialogue avec les experts, de doter le citoyen de sens critique et d'un pouvoir d'agir ${ }^{28}$.

C'est à ce type de considérations portées par l'ordinaire culturel des sciences et techniques auxquelles les étudiant-e-s sont invité-e-s à s'intéresser. Observer la façon dont ces rapports entre sciences, techniques et société sont vécus, représentés, perçus par les publics au travers de ses manifestations culturelles les plus ordinaires s'avère être un moyen judicieux d'aborder progressivement et autrement le champ STS, tout en partant de ce que les étudiant-e-s sont eux/elles mêmes. Pour se faire, ils/elles sont mise-s en contact avec quelques-unes de ses manifestations publiques les plus évidentes.

\section{2. Premiers regards collectifs} d'existence culturelle des sciences et techniques, telles qu'elles se donnent à voir au travers de la production et de la consommation de masse. Ils/elles sont ainsi projeté-e-s dans un espace public saturé de ces manifestations les plus anodines mais non moins significatives sur ce que les sciences et techniques font à la société, et inversement. Il suffit pour cela de partir d'un support, d'un texte ou d'une image partout présente dans l'espace public afin de susciter les premiers échanges. C'est ce qui a été fait par exemple cette dernière année, partant d'une des nombreuses affiches de campagne de l'association «vaincre la mucoviscidose", de celles qui occupent régulièrement les espaces publicitaires de la RATP et d'ailleurs ${ }^{29}$.

petit garçon le torse nu, serti d'un brun d'ADN métallique, demande avec son air tristounet de combattre avec lui cette maladie d'origine génétique et de faire un don. La parole est aux étudiant-e-s. Que voyez-vous ? Décrivez. A partir de la parole déployée, une multitude de questions émerge. Pourquoi est-ce une association de patients qui a en charge le financement de ce type de recherche? Le dogme central de la biologie est-il aussi dur que le fer de la molécule d'ADN figurée sur cette affiche? Quels sont les ordres de priorité en matière de recherche sur les maladies génétiques et pourquoi ? Quel est le rôle des patients dans l'organisation de la recherche? Quel est celui de l'industrie pharmaceutique et de l'État? Que s'agit-il au juste de découvrir et de mettre au point ? Le dépistage et le diagnostic anténatal sont-ils pertinents? S'ébauchent ainsi des questionnements sur la production conjointe de la maladie et de la société dans laquelle la mucoviscidose devient sur cette affiche une lutte mobilisant les publics. De là surtout le but de susciter parmi les étudiant-e-s le désir d'approfondir ces questions à partir de la lecture de travaux STS suggérés à l'issue de cette première séance ${ }^{30}$.

Cet exercice partant d'une description collective est un point d'entrée vers la compréhension de ce que sont les STS et de leur pertinence d'analyse pour la formation à la médiation. Car décrire, c'est commencer à comprendre ${ }^{31}$. C'est tout au moins s'engager sur la voie de l'émancipation intellectuelle ${ }^{32}$. Aussi cet exercice d'interprétations des 
œuvres plus ou moins informelles de la culture ordinaire se poursuit, mais toujours partant de documents imposés par l'enseignant.

Parmi quelques exemples, le tableau de Joseph Wright of Derby de 1768 intitulé «Expérimentation sur un oiseau dans une pompe à air » a été proposé pour susciter les commentaires sur la mise en scène des savoirs et des publics. On y voit une démonstration expérimentale parmi une audience bourgeoise où chaque personnage incarne une fonction sociale singulière en lien avec la production des savoirs scientifiques. Là encore surgissent des questions contemporaines de nature STS, quand bien même ce tableau parle à plus de deux siècles de distance. La suggestion de lire l'incontournable ouvrage de Steven Shapin et Simon Schaffer suit immanquablement ${ }^{33}$.

Que dire ensuite de la comparaison des tableaux des leçons d'anatomie du professeur Tulp d'après Rembrandt van Rijn (1632) et du Docteur Velpeau selon François-Nicolas Feyen-Perrin (1860)? Et que dire des tableaux de Georges Chicotot (1868-1921)? Comment rendre compte de leur pérennité dans nos imaginaires aujourd'hui jusqu'à les retrouver illustrant des manuels scolaires ou inspirer le travail d'artistes contemporains?

La peinture n'est évidemment pas la seule manifestation culturelle des sciences et techniques en société. Il y a beaucoup à dire partant d'un extrait du texte de LouisSébastien Mercier publié en 1782 - le Tableau de Paris - dans lequel il restitue à sa façon le lien permanent des arts, de l'industrie et de la vie quotidienne dans les recoins de la Capitale. Un extrait d'Honoré Balzac puisé dans $L a$ recherche de l'absolu permet de problématiser une épistémologie qui résonne étrangement avec les interrogations STS contemporaines. Que dire des romans scientifiques de Maurice Renard au début du XX siècle? Que tirer de la description du dialogue graphique entre Arago et Pasteur sur un billet de 500 francs en 1927 ? Que conclure de l'arrière-fond industriel des affiches de la campagne présidentielle de Valery Giscard-d'Estaing en 1981 ?

Arrêtons là les exemples. Beaucoup d'autres documents sont de cette façon utilisés afin de familiariser les étudiant-e-s à la fois à la culture ordinaire des sciences et techniques en société et à la problématisation de leurs analyses en termes STS. D'abord conduit en groupes, ce travail permet de confronter les points de vue, de lister les thèmes à caractère STS que les étudiant-e-s repèrent. Ils/elles ne font cependant à ce stade qu'interpréter spontanément des documents imposés, sans possibilité de recherche personnelle ni documentation. L'étape suivante consiste au contraire à les mettre seul-e-s à l'épreuve de l'immensité du champ de la culture ordinaire des STS afin d'aiguiser leurs capacités d'observation, d'analyse et de synthèse.

\section{3. Identifier ce qui fait sens}

40 Chaque étudiant-e s'engage à ce moment-là de l'année (à mi-parcours de la formation) dans l'exploration de ce qui fait sens pour lui/elle parmi la production foisonnante de la culture ordinaire. Publicité, fiction cinématographique, chantier de construction, chanson populaire, jeux vidéo ou œuvre de street art - peu importe comment les STS se manifestent dans l'ordre culturel du quotidien, dans le passé et aujourd'hui - la justification de focaliser l'attention sur telle expression singulière est qu'elle dise quelque chose des rapports entre sciences, techniques et société.

41 Ce qui suppose d'entendre ce qui se dit au travers de la culture ordinaire, de ne pas s'interdire le dialogue avec les publics. Il suffit de rappeler que la légèreté permet parfois 
de transmettre les messages les plus profonds. Une comédie ou un slogan publicitaire peut renvoyer plus sûrement à une conviction scientifique qu'un énoncé doctement délivré par un Nobel. Que cette conviction soit validée par les autorités scientifiques compétentes ou qu'elle soit une absurdité condamnée par l'Académie ne change rien. Son expression par les voies de la production et de la consommation culturelle de masse participe de fait d'une mise en culture de la science et de la technique dans ses rapports avec la société.

La science-fiction déploie par exemple des possibles enfouis dans le présent et cristallise des scenarii d'avenir ${ }^{34}$. En rendant sensibles des peurs et des espérances collectives, elle est toujours - à sa façon - politique. Elle interpelle, sert d'alerte ou d'exutoire des angoisses, bouscule les frontières entre les promesses rassurantes et la réalité du quotidien. Elle devient «science friction » quand elle orchestre la crainte d'une dérive technoscientifique menaçant le devenir de la planète ou de l'humanité.

Nombre des histoires les plus populaires de Michael Crichton peuvent être par exemple lues partant de cette grille de lecture, mêlant espoirs d'une vie meilleure et les angoisses d'une destruction par le déploiement non contrôlé de la technoscience. Dans The prey (2002), roman basé sur les suites catastrophiques de nanotechnologies échappées de leur laboratoire confiné, son auteur dit à la fois le potentiel extraordinaire du génie humain, tout en mettant en scène la question de ses capacités à en maîtriser les conséquences ${ }^{35}$. Le cinéma regorge ainsi de propositions du même genre contenant une science et une technique faisant culture.

Pour rester avec Michael Crichton, pensons à Jurassik park (1990), œuvre majeure de la culture populaire adaptée au cinéma par Steven Spielberg (1993), dans laquelle sont abordées de passionnantes questions en lien avec les aspects sociopolitiques de la science et de la technique. Le clonage est-il possible ? Pour quoi faire ? Est-ce dangereux ? A qui revient-il de contrôler ce qui se fait? Peut-on faire confiance dans ces domaines aux entreprises, aux gouvernements, aux militaires? Certes ici certaines des réponses suggérées dans ces œuvres seront à juste titre jugées extravagantes d'un point de vue strictement scientifique. Ce produit culturel de masse n'en aura pas moins popularisé la question du clonage et de ses impacts potentiels. Il aura suscité de véritables questions, peut-être même éveillé la curiosité, les vocations professionnelles et quelques consciences ... bref, il aura contribué à remplir nombre des fonctions supposées incomber aux dispositifs de la culture scientifique et technique telle que prescrite par les politiques publiques.

C'est en partant de la démonstration que l'analyse de ces manifestations de la culture ordinaire sont dans ce contexte de formation les conditions d'une heuristique des STS que les étudiant-e-s sont incité-e-s à la fréquenter assidument. Que ce soit sous la forme de discours, d'images, d'objets ou de représentations, ces témoignages font exister nombre de questionnements, de fantasmes et de promesses, d'espoirs et de craintes. Aux étudiant-e-s de les repérer, de les identifier, de les faire parler. Il leur est alors demandé de focaliser leur attention sur l'une de ces manifestations, quel que soit son mode d'expression. Autrement dit, ils/elles sélectionnent une trace, une archive, un objet, une image, bref ils/elles identifient un document considéré comme la traduction (textuelle ou graphique) de cette manifestation de la culture ordinaire en lien avec les questionnements à caractère STS d'une époque. Aucune limite n'est imposée sinon celle de justifier que ce choix témoigne de l'existence de cette culture ordinaire dont il s'agit de saisir la pertinence au regard des STS. 


\section{4. Exercice individuel d'analyse et de synthèse}

A partir de ce choix individuel, l'étudiant-e formule ses questionnements afin de rédiger un texte de 5000 signes dans lequel est exposé l'intérêt du document sélectionné au regard de la culture ordinaire et des STS. Il/elle en précise le statut comme archive. Il/ elle précise ce que ce document était dans le contexte de sa production et ce qu'il est devenu aujourd'hui quand il/elle en parle ici et maintenant. Le document doit ainsi constituer la base de sa réflexion. Il n'est donc pas une simple illustration du propos mais une matière première de travail. L'étudiant-e doit le faire parler. Il/elle a pour cela tout loisir de mobiliser la documentation nécessaire et d'user des outils de recherche à sa disposition. Chaque texte rend ainsi compte du choix de ce document au titre d'un témoignage significatif de la culture ordinaire des STS. Sa rédaction est toutefois d'une facture standardisée. Elle comprend un titre court évoquant autant que possible le thème STS exposé dans le texte; la date associée à l'archive ; l'image du document (textuel ou graphique) et sa légende ; 5000 signes d'explicitation du choix et d'interprétation de ce que dit ce document.

Une première version du texte peut-être proposée à la lecture de tou-te-s si l'étudiant-e le souhaite. Il suffit pour cela de le publier sur un site de réalisation pédagogique : la Lucarne ${ }^{36}$. Ce site ouvert et public a pour vocation de servir d'atelier collaboratif pour la réflexion collective sur la médiation culturelle des sciences et techniques en société. Son but est d'assurer les lectures croisées, les commentaires (y compris émanant de lecteurs anonymes), de motiver les questions et suggestions d'amélioration de la rédaction. Une version ultime du texte est défendue oralement en fin d'année par chacun des auteurs devant l'ensemble de la promotion lors d'une séance de discussion générale.

D'abord collective et informelle, l'interprétation des manifestations de la culture ordinaire des sciences et techniques est ensuite graduellement individualisé. L'exercice aboutit en fin de parcours à la production d'un texte dans le cadre de contraintes imposées. Son but est de stimuler une faculté de jugement personnel. C'est à un exercice d'analyse et de synthèse auquel sont de cette façon soumis-es les étudiant-e-s. Ils/elles sont ainsi initié-e-s à la lecture STS partant d'un document toujours disponible dans le champ infini de l'espace public, tout en étant familia-risé-e-s à une matière qui ne soit pas seulement celle des opérateurs habituels de la culture institutionnelle, mais telle qu'elle est appropriée par les publics eux-mêmes. Tel un passage entre culture prescrite et culture ordinaire des STS, l'exercice aboutit aussi à mettre en évidence, afin de mieux les surmonter, les tensions entre ces deux champs de la culture des sciences et techniques. Car il ne faut pas sous-estimer ce que ce type de démarche suppose de transgression de frontières entre cultures prescrites/normatives et représentations collectives, entre cultures élitaires et cultures populaires, entre différents ordres de la rationalité, et de façon générale entre de multiples réalités culturelles ${ }^{37}$.

\section{5. Les STS, un fait culturel total}

La variété des choix, des idées, des enthousiasmes et des regards est amplement discutée lors des séances de cours mais surtout de l'une à l'autre entre les élèves eux-mêmes. Ils éprouvent de cette façon que la production des sciences et les pratiques techniques sont des faits culturels totaux au fondement des sociétés modernes, qu'à l'évidence leur 
fabrication procède de ce culte de la modernité dont les ramifications sont à repérer jusque dans les gestes les plus ordinaires. La société est de toute façon pétrie et saturée de problématiques STS. L'espace public regorge de ces témoignages anodins comme les plus évidents de ces préoccupations, cultivant pêle-mêle le débat et la controverse, une vision éthérée de l'innovation et du progrès, l'espoir et la crainte, les idées fausses comme les vérités les plus établies. Ces témoignages se manifestent selon des formes les plus triviales comme les plus subtiles. Tous les supports de diffusion, tout ce qui fait "culture» est ainsi colonisé par ces représentations de ce que sont la science et la technique, de leur place et de leur fonction dans la quête d'une modernité et de maitrise de ses dérives. La société se les approprie à sa manière par des actes différenciés de création et d'expression, de consommation comme de défection. Il s'agit dès lors d'observer les STS telles qu'elles se donnent à voir au sein de l'immensité de la culture ordinaire, mais sans préjuger des intentions de faire ouvre de culture prescrite. Indépendamment des intentions de diffusion d'une culture consciente d'elle-même, une culture ordinaire s'approprie ainsi sans plus de revendication les espoirs, les fantasmes, les désirs et les angoisses suscités tant par la science et la technique, que par leurs implicites politiques ${ }^{38}$.

50 Certes, le meilleur comme le pire, tout se donne à voir au travers de cette culture ordinaire. Ses manifestations confortent l'angoisse comme les espoirs les plus fous. Entre névrose paranoïaque et béatitude naïve face aux promesses d'une technoscience édifiante, il y a place pour une multitude d'interrogations, de questionnements, de méfiances et de défiances légitimes face aux prescripteurs de la culture institutionnelle, face aux entreprises désireuses de faire à tout prix le bonheur de l'humanité et aux experts garantissant trop fermement la stabilité de leurs modèles performatifs ${ }^{39}$. La culture ordinaire renvoie ainsi toujours d'une façon ou d'une autre à des interrogations légitimes, qu'elles soient sombres et liées à des menaces, ou qu'elles soient au contraire associées aux convictions d'une vie meilleure grâce aux progrès de la science et de la technique ${ }^{40}$. Le spectre des assurances et des inquiétudes est large, signifiant une réalité vécue qui ne peut être ignorée au motif d'une inadéquation de leurs expressions avec les cadres plus formels de la culture prescrite, normative et institutionnelle. Que cette culture ordinaire idéalise le progrès ou l'innovation, ou qu'elle en dénonce leurs méfaits, elle interroge politiquement, en creux ou explicitement, la science et la technique, les experts, les autorités publiques, l'industrie et l'entreprise.

\section{6. Une culture STS transverse}

51 Le travail d'analyse collective et individuelle partant de l'ordinaire des STS invite, on l'a $\mathrm{vu}$, à investir la question des enjeux sociaux et politiques de la culture autrement qu'en lisant des textes de STS. En mettant les étudiant-e-s eux-mêmes en situation de publics ignorants devant un produit de la culture ordinaire, ils/elles exercent leur capacité à élaborer des ordres de pensée et du discours relevant pleinement des STS. Ils/elles réalisent que la culture ordinaire est un espace informel de dialogue pertinent entre les producteurs de savoirs experts et la masse des publics.

52 Au travers de l'exploration de la culture ordinaire, les étudiant-e-s se confrontent aux discussions sur les enjeux de la médiation aujourd'hui. D'autres regards s'ouvrent sur les fonctions sociopolitiques de la médiation, par exemple par une interrogation sur le sens et la pertinence d'une communication édifiante des sciences et de la prouesse technique ${ }^{41}$. 
Car il s'agit désormais moins d'apprendre à faire aimer les sciences et les techniques pour elles-mêmes que de comprendre la complexité de leurs intrications avec le social, de repenser le dialogue démocratique ${ }^{42}$.

53 Cette façon d'appréhender les STS par le détour de la culture ordinaire questionne la posture du médiateur en général, et singulièrement celle de chacun et chacune dans cette formation. La distance trop affirmée avec les publics au début du parcours est ainsi reconsidérée, de même la démarcation entre la culture prescrite et la culture ordinaire. La première a certes des ambitions louables et bienveillantes, mais elle demeure normative. Elle fixe les cadres légitimes de l'énonciation des savoirs et de leur réception, disqualifiant en retour d'autres formes d'expression culturelle ${ }^{43}$. A contrario, la culture ordinaire suggère une réappropriation des sciences et techniques par la production et la consommation culturelle de masse. Elle permet finalement d'observer la façon dont s'opère l'acculturation de tous et toutes aux sciences et techniques en société.

À rebours donc d'une culture prescrite fixant les cadres et contenus de ce qui doit être su ou interrogé, les étudiant-e-s investissent les imaginaires et les interrogations tacites sur les liens entre sciences, techniques et société. Ils/elles empruntent les voies transverses de nos représentations que supportent la production et la consommation culturelle de masse ${ }^{44}$. Ils/elles mettent un temps à distance pour mieux y revenir les questions habituelles posées depuis le champ de la culture prescrite - celles qui portent sur la pertinence des savoirs contenus dans les dispositifs de diffusion et sur l'efficacité de leur réception - pour interroger l'ordinaire des sciences et des techniques, et ce qu'il recèle de questionnements sur leurs fonctions sociales et politiques dans un monde en devenir, espéré, fantasmé ou redouté. C'est finalement à un déplacement des regards auquel invite l'interprétation de la culture ordinaire, depuis la fabrication d'une culture confinée dans les lieux de sa production légitime vers l'expression d'une culture telle que les publics la vivent dans la réalité de leurs pratiques ordinaires.

\section{7. Réconcilier culture scientifique et cultures ordinaires des sciences}

55 La culture ordinaire constitue donc l'un de ces lieux où s'élaborent et se jouent les représentations communes sur ce que sont les sciences et les techniques en société. Aucun espace du social n'échappe à son influence. L'imprégnation du milieu scolaire est un exemple de cette dynamique constante de formation et de déformation de nos imaginaires par la culture ordinaire. Des images de la science et de la technique y sont en permanence remaniées, structurant les cadres formels et informels de la culture STS de masse. Les matières, les disciplines, les manières d'instruire sont à la fois des lieux de normalisation du savoir et des creusets pour la fabrication des imaginaires, tout comme le sont les productions de la culture ordinaire à laquelle les élèves sont soumis en permanence. Des images de la biologie exposées dans un manuel scolaire à celles auxquelles renvoie Jurassik park il y a certes bien des différences, mais toutes participent de cette médiation massive des sciences et techniques dans leurs relations avec la société.

Rien ici n'est neutre. Tout est partiel et partial. Tout est affaire de mise en scène et en récit au travers de cette culture vivante. Elle opère là sous nos yeux à chaque instant. Aux étudiant-e-s de la fréquenter pour éprouver des relations complexes que les sociétés modernes entretiennent avec la production des sciences et les pratiques techniciennes. Les pistes sont aussi nombreuses que le sont les voies de la mise en culture des sciences et 
techniques, variant les registres du formel et de l'informel. Que cette manifestation culturelle des sciences et des techniques en société soit intentionnelle ou tacite, considérée comme telle ou pas, peu importe. Ce qui compte est la lecture que l'on peut en faire. Il ne s'agit pas de chercher à savoir si les choses sont vraies ou même réalistes, mais d'interpréter ce qu'elles disent, non seulement en termes de contenu de savoirs/nonsavoirs mais surtout en termes de représentations de ce que nous sommes. Cela ne signifie pas que la vérité n'a plus aucune importance. Elle l'est assurément. La production de connaissances scientifiques comme l'efficacité technique restent les fondements d'une explication rationnelle du monde. En revanche cela signifie que les promesses et fantasmes portés par la production culturelle de masse sont pleins de sens sur la façon dont est pensé leur rôle dans nos sociétés, mais aussi leur influence sur nos identités comme êtres sociaux et humains.

Pour autant la formation à la médiation des STS ne délaisse pas la culture prescrite et institutionnelle. Seulement elle cherche à en préciser les contours, à interroger la légitimité de ses frontières conventionnelles et de ses performances au regard des objectifs que ce domaine d'intervention culturelle se fixe traditionnellement. Des allers et retours sont nécessaires tout au long de la formation afin de saisir ce qui fait culture et pourquoi. L'essentiel reste de concevoir la production culturelle tant du point de vue des producteurs eux-mêmes de savoirs et de performances techniques, que du point de vue ordinaire de la multitude que nous formons, des collectifs d'usagers que nous constituons, des consommateurs culturels que nous sommes, mais aussi des citoyens que nous prétendons incarner à chaque instant.

\section{Conclusion}

La culture des sciences et techniques en société c'est donc aujourd'hui tout cela: des dispositifs de médiation informelle clairement identifiés, mais aussi des savoirs et des imaginaires technoscientifiques contenus dans des objets, des messages, des usages, des discours, des images ou des textes les plus anodins et en apparence sans intérêt. Encore faut-il les faire parler. C'est à cet exercice d'interprétation auquel sont exercé-e-s les étudiant-e-s. Son but est de repousser l'horizon culturel dans ce qu'il a de non conventionnel, car l'extension du domaine de la culture des sciences et techniques n'a de limites que celles de notre capacité à en repérer les signes parmi le foisonnement de leurs manifestations. La culture ordinaire est ainsi fréquentée assidument tout au long de ce parcours de formation. Par ce détour s'inventent des documents et s'écrivent des textes. Entre objets intermédiaires et objets frontières ${ }^{45}$, ils forment les voies d'une transition pédagogique vers les STS pour la formation des futurs médiateur-rice-s.

L'expérience montre que le décentrage depuis la culture prescrite permet aux étudiant-es de s'approprier autrement les STS. C'est en se confrontant à la banalité de leurs manifestations qu'ils se les approprient. En empruntant cette voie de transition, ils/elles consentent à investir ce domaine en lien avec la médiation et leurs publics. Nombreux-ses acceptent finalement de lire les travaux STS, ceux que leur confrontation personnelle à la culture ordinaire suggère, guidé par l'enseignant. Nul besoin donc de focaliser l'attention sur la lecture critique des grands textes, tout au moins dans un premier temps. La voie empruntée suggère d'autres manières de lire, de voir, de dire les STS. Non pour abandonner les textes essentiels, mais pour mieux finalement y venir. Enfin, la démarche valorise la culture ordinaire comme matière à partir de laquelle peuvent se pratiquer 
d'autres formes de médiation des STS : entre les enseignants et les étudiants, entre les étudiant-e-s eux/elles-mêmes, entre les médiateur-rice-s et les publics auxquels ils s'adressent.

\section{BIBLIOGRAPHIE}

Albe, V., (2009). Enseigner les controverses, Rennes, PUR, 2009.

Bensaude-Vincent, B., (2009). Les vertiges de la technoscience. Façonner le monde atome par atome, Paris, Éditions la découverte.

Bensaude-Vincent, B., (2013). L'opinion publique et la science. A chacun son ignorance, Paris, Éditions la découverte.

Bensaude-Vincent, B., (2014). «The politics of buzzwords at the interface of technoscience, market and society: The case of "public engagement in science" », Public Understanding of Science, tome $23, \mathrm{n}^{\circ} 3$, p. $238-253$.

Bonneuil C. et al. (dir.) (2013). Une autre histoire des « Trente Glorieuses ». Modernisation, contestations et pollutions dans la France d'après-guerre, Paris, Editions la découverte.

Bonneuil, C. et Joly, P.-B. (2013). Sciences, techniques et société, Paris, Éditions la découverte Bourg, D. et Boy, D., (2005). Conférences de citoyens, mode d'emploi, Paris, Charles Léopold Mayer.

Bourg, D. et Whiteside, K., (2010). Vers une démocratie écologique : le citoyen, le savant et le politique, Le Seuil/La République des idées.

Boy, D., (1999). Le progrès en procès, Paris, Presses de la renaissance.

Bruno, I. (2008), À vos marques ${ }^{\oplus}$, prêts... cherchez ! La stratégie européenne de Lisbonne, vers un marché de la recherche, Bellecombes-en-Bauges, Éditions du croquant.

Callon, M. et al. (2001) Agir dans un monde incertain. Essai sur la démocratie technique, Paris, Seuil.

Caune, J., (2013) Pour des humanités contemporaines. Science, technique, culture : quelles médiations?, Grenoble, PUG.

Chavot, Ph., et Masseran (dir.) A. (2010) « Dossier. (Re)penser les sciences et les techniques en Europe ", Questions de communication, tome 17, et notamment « Engagement et citoyenneté scientifique : quels enjeux avec quels dispositifs ? ", p. 81-106.

Chavot, Ph., et Masseran (dir.) A. (2013). « Les cultures des sciences en Europe (1). Dispositifs en pratique », Questions de communication, tome 18 et (2015). « Les cultures des sciences en Europe (2). Dispositifs, publics, acteurs, institutions », Questions de communication, tome 25.

Cheng, D., Metcalfe, J., Schiele, B., Claessens, M., Gascoigne, T., and Shunke, S., (2006). At the Human scale - International practices in science communication, Pékin, Science Press.

Claessens M., (2011) « Programmer la recherche : facile mais... impossible », Innovations tome 3, $\mathrm{n}^{\circ}$ 36 , p. 21-39.

Claessens, M., (1998). La Technique contre la démocratie, Paris, Seuil. 
Claessens, M., (2009). Science et communication : pour le meilleur ou pour le pire?, Versailles, Éditions Quae.

Convert, B. et Demailly, L., (2012) «Effets collatéraux de la création littéraire. L'exemple de la science-fiction ", Sociologie de l'Art, tome 21, n³, p. 111-133.

Felt, U. et al. (2007). Taking european knowledge society seriously, European Commission, Directorate-General for Research Science, Economy and Society.

Garçon, A.-Fr., (2013). L'Imaginaire et la pensée technique. Une approche historique, $X V I^{e}-X X^{e}$ siècle, Paris, Classiques Garnier.

Gervereau, L., (1994). Voir comprendre analyser les images, Paris, Éditions la découverte.

Hayat, S., (2017). «Les savoirs et leurs publics : l'exemple du Conservatoire des arts et métiers (XIX ${ }^{\mathrm{e}}-\mathrm{XXI}^{\mathrm{e}}$ siècles) », Innovations, tome 52, n 1, p. 139-160.

Hottois, G., (2006). «La technoscience : de l'origine du mot à ses usages actuels », Recherche en soins infirmiers, tome $86, \mathrm{n}^{\circ} 3$, p. 24-32.

Jacq, A., et Guespin-Michel, J., (2015). « Science et démocratie : une articulation difficile mais nécessaire », Ecologie et politique, tome 51, n², p. 107-120.

Jasanoff, S., (2014). «A mirror for science », Public Understanding of Science, tome 23, n¹, p. 21-26. Larqué Lionel, L. et Pestre, D. (dir.), (2013). Les sciences ça nous regarde. Histoires surprenantes de nos rapports aux sciences et aux techniques, Paris, Éditions la découverte.

Larqué, L., (2006). «Au chevet de la culture scientifique et technique », Alliage, tome 59, p. 10-19; Namur, D. et Paillard, S., (2006). « Science et communication : promettre ou éclairer », Hermès, $L a$ Revue, tome $44, \mathrm{n}^{\circ} 1$, p. 107-112.

Las Vergnas, O., (2011). «L'institutionnalisation de la "culture scientifique et technique", un fait social français $(1970-2010) »$, Savoirs, tome $27, n^{\circ} 3$, p. 9-60.

Lemieux, C. et Barthe, Y., (1998). « Les risques collectifs sous le regard des sciences du politique. Nouveaux chantiers, vieilles questions ", Politix, tome $44, \mathrm{n}^{\circ} 11$, p. 7-28

Lemieux, C., (2007). « À quoi sert l'analyse des controverses ? », Mil neuf cent. Revue d'histoire intellectuelle, tome $25, \mathrm{n}^{\circ} 1, \mathrm{p} .191-212$.

Lévy-Leblond, J.-M., (2014). « La culture scientifique, pourquoi faire ? », Alliage, tome 73, p. 17-30.

Olivier, M. et Leleux, J.-P., (2014). Faire connaître et partager les cultures scientifiques, techniques et industrielles : un impératif, rapport $\mathrm{n}^{\circ} 274$ : http://www.senat.fr/rap/r13-274/r13-2741.pdf

Pérez, L., (2009). « L'histoire intellectuelle des techniques au Centre d'histoire des techniques et de l'environnement du Conservatoire national des arts et métiers (Cdhte-Cnam) », Revue de synthèse, tome 130, p. 147-164.

Pestre, D., (2013), A contre-science. Politiques et savoirs des sociétés contemporaines, Paris, Seuil.

Quet, M., (2012). « La critique des technologies émergentes face à la communication promettante. Contestations autour des nanotechnologies ", Réseaux, tome 173-174, n³, p. 271-302.

Rabehariso, V. et Callon, M., (2002). « L'engagement des associations de malades dans la recherche ", Revue internationale des sciences sociales, tome $171, \mathrm{n}^{\circ} 1, \mathrm{p} .65-73$.

Rancière, J., (1987). Le maître ignorant. Cinq leçons sur l'émancipation intellectuelle, Paris, Fayard.

Reber, B., (2005). «Technologies et débat démocratique en Europe. De la participation à

l'évaluation pluraliste ", Revue française de science politique, tome $55, n^{\circ} 5$, p. 811-833. 
Schaffer, S. et Shapin, S., (1986). Léviathan et la pompe à air : Hobbes et Boyle entre science et politique (trad.), Paris, Éditions la découverte.

Stengers, I., (1997). Sciences et pouvoirs. La démocratie face à la technoscience, Paris, Éditions de la Découverte

Suraud, M.-G., (2013). « La contestation des "nanos" : redéfinir la notion de "politisation de la science" ", Les Enjeux de l'information et de la communication, tome 14, n¹, p. 117-129.

Topçu, T., (2013). « Technosciences, pouvoirs et résistances : une approche par la gouvernementalité ", Revue d'histoire moderne et contemporaine, tome 60-4, $\mathrm{n}^{\circ} 4$ bis, p. 76-96.

Trichet, Y. et Marion, E., (2014). « Le savant dans le malaise contemporain, entre désir et jouissance », Bulletin de psychologie, tome 531, n³, p. 225-235.

Vessuri, H., (2001). « Introduction : la science et ses cultures », Revue internationale des sciences sociales, tome $168, \mathrm{n}^{\circ} 2$, p. 199-206.

Vinck, D., (2009). « De l'objet intermédiaire à l'objet-frontière. Vers la prise en compte du travail d'équipement », Revue d'anthropologie des connaissances, tome $3, \mathrm{n}^{\circ} 1$, p. 51-72.

Winner, L., (2002). La baleine et le réacteur. A la recherche de limites au temps de la haute technologie, Paris, Descartes et Cie.

Wynne, B., (1992). « Misunderstood misunderstanding: social identities and public uptake of science ", Public Understanding of Science, tome 1, $\mathrm{n}^{\circ}$ 3, p. 281-304.

\section{NOTES}

1. Pérez, L., (2009). «L'histoire intellectuelle des techniques au Centre d'histoire des techniques et de l'environnement du Conservatoire national des arts et métiers (Cdhte-Cnam) », Revue de synthèse, tome 130, p. 147-164.

2. Hayat, S., (2017). « Les savoirs et leurs publics : l'exemple du Conservatoire des arts et métiers (XIXe-XXIe siècles) », Innovations, tome 52, n 1, p. 139-160.

3. Bruno, I. (2008), À vos marques ${ }^{\oplus}$, prêts... cherchez! La stratégie européenne de Lisbonne, vers un marché de la recherche, Bellecombes-en-Bauges, Éditions du croquant.

4. "Conclusion de la présidence. Conseil européen de Lisbonne, 23 et 24 mars 2000 », http:// www.consilium.europa.eu/fr/uedocs/cms_data/docs/pressdata/fr/ec/00100-r1.fo.htm

5. Philippe Chavot, Ph., et Masseran (dir.) A. (2013). « Les cultures des sciences en Europe (1). Dispositifs en pratique ", Questions de communication, tome 18 et (2015). " Les cultures des sciences en Europe (2). Dispositifs, publics, acteurs, institutions », Questions de communication, tome 25 .

6. Felt, U. et al. (2007). Taking european knowledge society seriously, European Commission, Directorate-General for Research Science, Economy and Society.

7. Lemieux, C., (2007). « À quoi sert l'analyse des controverses? », Mil neuf cent. Revue d'histoire intellectuelle, tome $25, \mathrm{n}^{\circ} 1$, p. 191-212.

8. Sur les usages du terme "technoscience ", voir la mise au point de Hottois, G., (2006). « La technoscience : de l'origine du mot à ses usages actuels ", Recherche en soins infirmiers, tome 86, $\mathrm{n}^{\circ} 3$, p. 24-32.

9. Stengers, I., (1997). Sciences et pouvoirs. La démocratie face à la technoscience, Paris, Éditions de la découverte ; Lemieux, C. et Barthe, Y., (1998). "Les risques collectifs sous le regard des sciences du politique. Nouveaux chantiers, vieilles questions ", Politix, tome 44, n¹1, p. 7-28 ; 
Reber, B., (2005). «Technologies et débat démocratique en Europe. De la participation à l'évaluation pluraliste ", Revue française de science politique, tome 55, n5, p. 811-833.

10. Bourg, D. et Whiteside, K., (2010). Vers une démocratie écologique : le citoyen, le savant et le politique, Le Seuil/La République des idées ; Claessens Michel, «Programmer la recherche : facile mais... impossible ", Innovations 3/2011 (n³6), p. 21-39 ; Callon, M. et al. (2001) Agir dans un monde incertain. Essai sur la démocratie technique, Paris, Seuil ; Bourg, D. et Boy, D., (2005). Conférences de citoyens, mode d'emploi, Paris, Charles Léopold Mayer.

11. Jacq, A., et Guespin-Michel, J., (2015). «Science et démocratie : une articulation difficile mais nécessaire ", Ecologie et politique, tome 51, n², p. 107-120 ; Topçu, T., (2013). « Technosciences, pouvoirs et résistances : une approche par la gouvernementalité », Revue d'histoire moderne et contemporaine, tome 60-4, $\mathrm{n}^{\circ} 4$ bis, p. 76-96.

12. Albe, V., (2009). Enseigner les controverses, Rennes, PUR, 2009 ; Winner, L., (2002).

13. Ce terme STS est employé par la suite pour désigner tant les thématiques ayant trait à ce champ d'étude que l'interprétation des dynamiques de co-construction des savoirs et de l'organisation sociale et politique. Pour une synthèse des approches dites STS, voir Bonneuil, C. et Joly, P.-B. (2013). Sciences, techniques et société, Paris, Éditions de la découverte.

14. Philippe Chavot, Ph., et Masseran (dir.) A. (2010). « Dossier. (Re)penser les sciences et les techniques en Europe ", Questions de communication, tome 17, et notamment « Engagement et citoyenneté scientifique : quels enjeux avec quels dispositifs? », p. 81-106.

15. Suraud, M.-G., (2013). « La contestation des "nanos" : redéfinir la notion de "politisation de la science" », Les Enjeux de l'information et de la communication, tome 14, n¹, p. 117-129.

16. Lévy-Leblond, J.-M., (2014). « La culture scientifique, pourquoi faire ? », Alliage, tome 73 ,

p. 17-30.

17. Pour une présentation plus détaillée de la formation en question, voir dans ce dossier : Petitgirard, L., (2018). « Hacker et collaborer : dispositifs pour la formation des médiateurs culturels (des sciences et techniques)».

18. Bensaude-Vincent, B., (2013). L'opinion publique et la science. A chacun son ignorance, Paris, Éditions la découverte.

19. Larqué Lionel, L. et Pestre, D. (dir.), (2013). Les sciences ça nous regarde. Histoire surprenantes de nos rapports aux sciences et aux techniques, Paris, Éditions la découverte.

20. Pestre, D., (2013), A contre-science. Politiques et savoirs des sociétés contemporaines, Paris, Seuil.

21. Bonneuil et al. (dir.) (2013). Une autre histoire des "Trente Glorieuses ». Modernisation, contestations et pollutions dans la France d'après-guerre, Paris, Editions la découverte.

22. Boy, D., (1999). Le progrès en procès, Paris, Presses de la renaissance.

23. Cheng, D., Metcalfe, J., Schiele, B., Claessens, M., Gascoigne, T., and Shunke, S., (2006). At the Human scale - International practices in science communication, Pékin, Science Press.

24. Wynne, B., (1992). « Misunderstood misunderstanding: "social identities and public uptake of science" ", Public Understanding of Science, tome 1, n 3, p. 281-304.

25. Las Vergnas, O., (2011). «L'institutionnalisation de la "culture scientifique et technique", un fait social français $(1970-2010) »$, Savoirs, tome $27, n^{\circ} 3$, p. 9-60.

26. Non sans ambiguïtés : voir par exemple Bensaude-Vincent, B., (2014). "The politics of buzzwords at the interface of technoscience, market and society: The case of "public engagement in science" ", Public Understanding of Science, tome 23, n³, p. 238-253.

27. L'un des derniers bilans officiels produit sur ces questions - celui de l'OPECST - assume et revendique clairement cette orientation : Olivier, M. et Leleux, J.-P., (2014). Faire connaître et partager les cultures scientifiques, techniques et industrielles : un impératif, rapport $\mathrm{n}^{\circ} 274$ : http://www.senat.fr/rap/r13-274/r13-2741.pdf 
28. Claessens, M., (1998). La Technique contre la démocratie, Paris, Seuil.

29. Cette affiche a été choisi de façon opportuniste parce qu'elle sature l'espace public à la veille de la séance de cours en question. Une autre publicité, l'affiche d'un film ou la couverture d'un ouvrage aurait tout aussi bien pu être envisagé.

30. Sont ainsi suggérées les lectures de Juven, P.-A., (2014). "Vaincre la mucoviscidose et maîtriser la tarification à l'activité. Un cas d'activisme gestionnaire », Participations, tome $9, \mathrm{n}^{\circ} 2$, p. 239-262 ; Houzard, S. et Routelous, C. (2014). " L'institutionnalisation de la mucoviscidose : histoire d'un réseau sociotechnique clinique abouti ", Quaderni, tome 85, n³, p. 53-66 ; Huyard, C., (2011). « Pourquoi s'associer? Quatre motifs d'entrée dans un collectif dans les associations de maladies rares ", Revue française de sociologie, tome 52, n 4, p. 719-745 ; Dalgalarrondo, S., (2007). "Quelle place pour les associations de malades dans le processus d'innovation médicamenteuse ? Le cas des maladies rares ", Revue française des affaires sociales, tome $3, \mathrm{n}^{\circ}$ 3-4, p. 171-191 ; Rabehariso, V. et Callon, M., (2002). «L'engagement des associations de malades dans la recherche ", Revue internationale des sciences sociales, tome 171, n¹, p. 65-73.

31. Gervereau, L., (1994). Voir comprendre analyser les images, Paris, Éditions la découverte.

32. Rancière, J., (1987). Le maître ignorant. Cinq leçons sur l'émancipation intellectuelle, Paris, Fayard.

33. Schaffer, S. et Shapin, S., (1986). Léviathan et la pompe à air : Hobbes et Boyle entre science et politique (trad.), Paris, Éditions la découverte.

34. Convert, B. et Demailly, L., (2012) «Effets collatéraux de la création littéraire. L'exemple de la science-fiction », Sociologie de l'Art, tome 21, n³, p. 111-133.

35. Crichton, M., (2004) La proie (traduction de Berthon, P.), Paris, Pocket.

36. http://ateliercst.hypotheses.org/

37. Vessuri, H., (2001). "Introduction : la science et ses cultures ", Revue internationale des sciences sociales, tome $168, \mathrm{n}^{\circ} 2, \mathrm{p} .199-206$.

38. Trichet, Y. et Marion, E., (2014). « Le savant dans le malaise contemporain, entre désir et jouissance », Bulletin de psychologie, tome 531, n³, p. 225-235.

39. Quet, M., (2012). « La critique des technologies émergentes face à la communication promettante. Contestations autour des nanotechnologies », Réseaux, tome 173-174, $\mathrm{n}^{\circ} 3$,

p. 271-302.

40. Bensaude-Vincent, B., (2009). Les vertiges de la technoscience. Façonner le monde atome par atome, Paris, Éditions la découverte.

41. Caune, J., (2013) Pour des humanités contemporaines. Science, technique, culture : quelles médiations ?, Grenoble, PUG ; Claessens, M., (2009). Science et communication : pour le meilleur ou pour le pire?, Versailles, Éditions Quae.

42. Larqué, L., (2006). « Au chevet de la culture scientifique et technique », Alliage, tome 59 ,

p. 10-19 ; Namur, D. et Paillard, S., (2006). «Science et communication : promettre ou éclairer », Hermès, La Revue, tome $44, \mathrm{n}^{\circ} 1, \mathrm{p} .107-112$.

43. Jasanoff, S., (2014), «A mirror for science », Public Understanding of Science, tome $23, n^{\circ} 1, p$. 21-26.

44. Garçon, A.-Fr., (2013). L'Imaginaire et la pensée technique. Une approche historique, XVIeXXe siècle, Paris, Classiques Garnier.

45. Vinck, D., (2009). « De l'objet intermédiaire à l'objet-frontière. Vers la prise en compte du travail d'équipement», Revue d'anthropologie des connaissances, tome 3, $n^{\circ} 1$, p. 51-72. 


\section{RÉSUMÉS}

La culture ordinaire, une heuristique des STS pour former à la médiation culturelle des sciences et techniques en société. Avec l'inscription à l'agenda européen de l'économie de la connaissance et du dialogue civil, la diffusion de la culture scientifique, des techniques et de l'industrie (CSTI) s'est progressivement tournée vers une médiation des questions dites socialement vives et des controverses sociotechniques. La formation dans ces domaines comprend désormais une forte composante STS, c'est-à-dire l'étude de la fabrication conjointe des sciences, des techniques et de la société. Au Conservatoire national des arts et métiers (Cnam), les enseignements destinés aux médiateur-rice-s ont intégré cette dimension, en partie sous la forme d'une démarche pédagogique dont le principe est l'analyse de l'expression de ces questions au travers de la culture ordinaire des sciences et techniques en société. Cette dernière comprend l'ensemble des médiations informelles repérables au sein de la production, des pratiques et de la consommation culturelle de masse. L'exercice qui consiste à les identifier et à les interpréter constitue pour les élèves une heuristique des STS. Cet article décrit l'expérience engagée afin que les étudiant-e-s découvrent par eux/elles-mêmes ce qui leur est enseigné : l'intrication complexe des sciences, des techniques et de la société dont la culture ordinaire est un des modes d'existence. La culture ordinaire est ainsi à la fois explorée comme espace, matériau, ressource et ultimement comme voie d'une transition pédagogique vers les questionnements STS en lien avec les pratiques professionnelles et les publics des médiateur-rice-s.

The knowledge economy and public debates are now part of the European political agenda. As a result, public understanding of science and technology as a culture has shifted to public engagement with science and technology. Science and Technology Studies (STS) is now an essential element of training in this field, considering science and society as a common production process. At the National Conservatory of Arts and Crafts (Cnam) in Paris, the pedagogical subjects dedicated to training in cultural activities of scientific and technological mediation integrate this dimension STS. To do this, students are invited to experience the relationship between science and technology and society through the analysis of ordinary culture. Ordinary culture is here the informal way in which STS questioning exists permanently through popular expressions, cultural products and acts of mass consumption. Part of the method is to identify and explain these popular expressions. This results in a sort of STS heuristic for the students. This article describes the experience of being involved in the process of selfunderstanding of what is at stake: the entanglement of science and technology and society. Ordinary culture is one of their most obvious translations. It is therefore not a question of considering ordinary culture solely as a subject, a space or a resource for the mediation of scientific culture, but also as a pedagogical means of training students in cultural practices directly related to STS questions. 
INDEX

Mots-clés : culture scientifique et technique, médiation culturelle, sciences et techniques en société (STS), culture ordinaire, transition pédagogique, culture informelle, Cnam

Keywords : scientific and technical culture, cultural mediation, science and technology in society, ordinary culture, pedagogical transition, informal culture, National Conservatory of Arts and Crafts (Cnam)

\section{AUTEUR}

\section{MICHEL LETTÉ}

Maître de conférences en histoire des techniques et de l'environnement, Conservatoire national des arts et métiers (CNAM) à Paris. 